\title{
Educação em saúde à pessoa com diabetes mellitus no hospital
}

\author{
Health education for people with diabetes mellitus in the hospital
}

Caroline Rocha Batista Barcellos ${ }^{1}$

Orcid: https://orcid.org/0000-0003-2793-0163

Bárbara Resende Ramos ${ }^{3}$

Orcid: https://orcid.org/0000-0001-9855-5052

Franciele Roberta Cordeiro 5

Orcid: https://orcid.org/0000-0001-6194-5057

\author{
Juliana Graciela Vestena Zillmer ${ }^{2}$ \\ Orcid: https://orcid.org/0000-0002-6639-8918 \\ Daniela Blank Barz ${ }^{4}$ \\ Orcid: https://orcid.org/0000-0001-5472-685X \\ Marina Borges Luiz \\ Orcid: https://orcid.org/0000-0003-1317-5355
}

\begin{abstract}
Resumo
OBJETIVO: Descrever as ações de enfermeiros para a educação em saúde da pessoa com diabetes mellitus hospitalizada. MATERIAIS E MÉTODOS: Pesquisa qualitativa, realizada em hospital de ensino no Sul do Brasil. Participaram 15 enfermeiros por amostragem intencional, que atuavam nos setores de clínica médica, clínica cirúrgica e redes de urgência e emergência. Os dados foram produzidos entre setembro e novembro de 2019, por meio de entrevista semiestruturada, gerenciados pelo programa Ethnograph v6 e submetidos à análise de conteúdo. RESULTADOS: Foram construídas cinco categorias, sendo elas: Orientar e se (re)adequar: evitando complicações; Educação para o cuidado com os pés e com a pele; Orientações quanto ao uso dos fármacos; Cuidados com a alimentação e Educação em saúde com os familiares. Os enfermeiros consideram a prática de educação em saúde no hospital como importante e, o processo educativo dos enfermeiros realizado contemplava ações que abrangeram as pessoas com diabetes mellitus e familiares. CONCLUSÕES: O enfermeiro, no hospital, pode impulsionar ações incluindo a implementação de grupos, criando espaço potencializado para compartilhar orientações e experiências de vida de pessoas com diabetes e o modo como cuidam da saúde.
\end{abstract}

Palavras-chave: doenças não transmissíveis; adulto; educação em saúde; pesquisa qualitativa.

\section{Abstract}

OBJECTIVE: To describe the actions of nurses in favor of health education for people hospitalized with diabetes mellitus. MATERIALS AND METHODS: this is a qualitative research, carried out in a teaching hospital in southern Brazil. The intentional sampling was 15 nurses who worked in the sectors of medical clinic, surgical clinic and urgent and emergency networks. Data were produced using semi-structured interviews from September to November in 2019 and managed subsequently by the Ethnograph v6 program, after that, they were submitted to content analysis. RESULTS: Five categories were identified, namely: Guide and (re) adapt: avoiding complications; Education for the care of the feet and skin; Guidelines on the use of drugs; Food care and health education with family members. Nurses claim the health education inside the hospital has a key role and their educational process has included some actions that covered both people with diabetes mellitus and their families. CONCLUSIONS: The nurse in the hospital can promote actions including the implementation of some groups for people with diabetes by creating a potentialized place in order to share guidelines and life experiences and to discuss the way they take care of their health.

Keywords: noncommunicable diseases; adult; health education; qualitative research.

\footnotetext{
${ }^{1}$ Hospital Regional Hans Dieter Schmidt. E-mail: caroline.rbb@gmail.com

${ }^{2}$ Universidade Federal de Pelotas. E-mail: juzillmer@gmail.com

${ }^{3}$ Universidade Federal de Pelotas. E-mail: barbararesende.ramos@gmail.com

${ }^{4}$ Universidade Federal de Pelotas. E-mail: danielabarzsls@hotmail.com

${ }^{5}$ Universidade Federal de Pelotas. E-mail: franciele.cordeiro@ufpel.edu.br

${ }^{6}$ Universidade Federal de Pelotas. E-mail: marinaborges_mari@hotmail.com
} 


\section{Introdução}

A diabetes mellitus destaca-se entre as doenças crônicas não transmissíveis pela crescente incidência, prevalência e morbimortalidade $o$ que a faz ser considerada um importante problema de saúde pública no cenário nacional e mundial. Estima-se que hoje, no mundo, 387 milhões de pessoas vivam com a doença, com expectativa de 471 milhões para 2035, principalmente adultos e idosos $1,2,3$.

No Brasil, no período de 2015 a 2019, ocorreram 668.111 internações por diabetes mellitus ${ }^{4}$. Trata-se de uma enfermidade responsável por inúmeras complicações, dentre elas, cardiopatias, neuropatia, nefropatia e doenças oculares, ocasionando um impacto econômico aos sistemas de saúde por apresentar maiores índices de internação e maior tempo de hospitalizações ${ }^{2,3,5}$. Diante do apresentado é necessário priorizar ações relacionadas à promoção da saúde e à prevenção de complicações, tornando a educação em saúde fundamental no contexto de assistência hospitalar ${ }^{6,7}$.

A educação em saúde se torna essencial e potencializa o autocuidado e a qualidade de vida das pessoas com diabetes e seus familiares ${ }^{6,8}$, e, consequentemente reduz as complicações ${ }^{6}$. Considera-se educação em saúde o "conjunto de práticas que contribui para aumentar a autonomia das pessoas no seu cuidado e no debate com os profissionais e os gestores a fim de alcançar uma atenção de saúde de acordo com suas necessidades"9,19. A partir dela a pessoa aprende a cuidar de sua saúde, que é decorrente de múltiplos fatores inerentes ao processo saúde-doença, a partir do conhecimento de sua realidade ${ }^{10}$. Ela é uma estratégia fundamental para o cuidado, uma vez que facilita o aprendizado, promove o conhecimento das pessoas para que aprimorem a capacidade de intervenção sobre suas vidas ${ }^{11}$.
O enfermeiro pode ser considerado um educador e, a educação em saúde uma estratégia de intervenção inerente à profissão ${ }^{12}$. Como membro da equipe multidisciplinar, no cuidado à pessoa com diabetes, ele tem papel essencial na promoção da saúde e prevenção de agravos, mediante a educação de pessoas com risco de complicações decorrentes de sua condição de saúde ${ }^{5}$. Trata-se de um profissional com competências e habilidades capazes de promover autonomia e empoderamento dos indivíduos no hospital ${ }^{17,12,13}$.

Contudo, ao consultar a literatura evidenciou-se que são incipientes os estudos sobre a educação em saúde no hospital $^{17,12}$ e, ainda menos os estudos acerca de educação em saúde a pessoas com diabetes nesse cenário ${ }^{13,14}$. A educação em saúde, desenvolvida no hospital, representa uma tendência emergente na enfermagem ${ }^{12}$. Nesse sentido, evidencia-se a necessidade de estudos desenvolvidos com essa população, que sirva como subsídio para ações futuras na perspectiva da promoção do autocuidado e na qualidade da assistência hospitalar. Diante do apresentado, a questão de pesquisa que norteou o estudo foi: Quais as ações dos enfermeiros para a educação em saúde da pessoa com diabetes mellitus hospitalizada? A partir do exposto, o presente estudo teve como objetivo descrever as ações de enfermeiros para a educação em saúde da pessoa com diabetes mellitus hospitalizada.

\section{Materiais e Métodos}

\section{Amostra e tipo de estudo}

Estudo de abordagem qualitativa, do tipo descritivo. Justifica-se a escolha desta abordagem de pesquisa, pois ela possibilita estudar o fenômeno em sua intensidade e por estar preocupada com os aspectos socioculturais que se expressa mediante relações, valores, crenças, simbologias, comportamentos, opiniões, representações e práticas além de compreender aspectos da 
realidade e contexto. Nesse sentido, a pesquisa qualitativa responde a questões particulares da experiência dos indivíduos em sua total magnitude, o que corresponde a um espaço mais profundo das relações, dos processos e dos fenômenos ${ }^{15}$.

\section{Delineamento da pesquisa}

Estudo realizado em unidades de internação adulto de um Hospital de Ensino do Rio Grande do Sul, Brasil. O Hospital possui 175 leitos, presta atendimento exclusivamente pelo Sistema Único de Saúde a 28 municípios. A escolha por tal Hospital justifica-se pelo mesmo ter vínculo com um Centro de Diabetes e Hipertensão, referência em atendimento à pessoa com diabetes na Região, e, ambos sob a responsabilidade de uma Universidade Pública. A produção de dados ocorreu entre setembro e outubro de 2019.

\section{Critérios de Inclusão e Exclusão}

Os participantes do estudo

foram enfermeiros, escolhidos por amostragem intencional, mediante os seguintes critérios de inclusão: ser enfermeiro da instituição há mais de seis meses e, ter vivenciado cuidados ao paciente com diabetes mellitus. O critério de exclusão foi estar em licença saúde ou férias. Desses 15 participantes, 13 do sexo feminino e dois do sexo masculino. A coleta de dados e o tamanho da amostra foram subsidiados a partir da proposta de saturação de informações ${ }^{15}$. Desta forma, na medida em que a informação coletada se repetia eram retiradas do campo de pesquisa e, paralelamente ocorria a construção de um esquema teórico para responder o objeto em estudo.

\section{Procedimentos}

Utilizou-se a entrevista semiestruturada como técnica de produção de dados, sendo desenvolvido um guia, contendo perguntas abertas e fechadas, buscando responder o objetivo da pesquisa. A primeira pergunta foi aberta, sendo ela,
"Podes me contar sobre sua experiência ao cuidar de pessoas com diabetes durante a hospitalização?", posteriormente seguida de outras que gradativamente possibilitaram aprofundar o tema. Esse instrumento continha perguntas que oportunizaram os profissionais falarem sobre experiências que tiveram com o cuidado dos pacientes com diabetes mellitus. Os cuidados que eram realizados e, as ações e orientações fornecidas durante a hospitalização. Além disso, em que momento a família participava do cuidado a esse paciente durante a internação e quais facilidades e dificuldades que os enfermeiros vivenciavam para cuidar desses pacientes.

As entrevistas foram realizadas individualmente, em horários previamente definidos com os participantes em uma sala reservada do hospital, conforme escolha do participante. Essas foram gravadas com gravador digital, a fim de permitir transcrição literal do conteúdo de áudio, sendo esta realizada pela primeira autora do estudo.

Os dados oriundos das entrevistas foram gerenciados pelo Programa Ethnograph V6 na versão demonstração e submetidos à análise de conteúdo proposta por Laurence Bardin a qual compreende as etapas de pré-análise, exploração do material, tratamento dos resultados e interpretações ${ }^{16}$. Tais etapas, neste estudo, ocorreram da seguinte forma: inicialmente, as entrevistas foram transcritas semanalmente, conforme a ordem de realização, pela primeira autora, no editor de texto Microsoft Word. Ao final dessa etapa, foi efetuada leitura flutuante das entrevistas e, posteriormente indexadas no Programa Ethnograph V6. A partir disso, a leitura se deu de forma criteriosa, a fim de identificar os pontos chaves da discussão do participante, sendo elaborados códigos. Em seguida, os códigos foram comparados e, fragmentos de texto selecionados para identificação dos temas, que posteriormente deram origem às categorias. Foram criados 24 códigos, e a partir destes foram construídas cinco categorias. 
Para garantir o anonimato dos participantes utilizou-se a primeira letra de Enfermeiro - E; seguido da ordem de realização das entrevistas, utilizando-se números cardinais $(01,02,03 \ldots)$. Diante do apresentado, os participantes foram denominados, por exemplo, da seguinte forma: Enfermeiro, entrevista 01 - E01.

Esta pesquisa seguiu a Resolução $n^{0}$ 466 de dezembro de 2012 do Conselho Nacional de Saúde, que diz respeito aos aspectos éticos envolvendo seres humanos, visando garantir os direitos e deveres dos participantes da pesquisa, comunidade científica e o Estado ${ }^{17}$. Obteve-se a aprovação pelo Comitê de Ética em Pesquisa de uma Universidade pública sob CAAE de número 19579519.8.0000.5317. Além disso, também respeitou os princípios do Código de Ética dos Profissionais de Enfermagem aprovado pela Resolução $564 / 2017{ }^{18}$. Todos os participantes assinaram o Termo de Consentimento Livre e Esclarecido

\section{Resultados}

Os participantes do estudo foram 15 enfermeiros, 13 do sexo feminino e dois do sexo masculino, com idades entre 31 a 47 anos. O tempo de atuação no hospital em estudo variou de um a quatro anos e o tempo de formação de dois a 23 anos. Em se tratando de especialização correspondem às áreas de Terapia Intensiva; Cuidados Paliativos; Atenção Psicossocial; Saúde da mulher, criança e adolescente; Saúde Mental; Saúde Coletiva; Saúde do Idoso; Oncologia; Auditoria em Saúde; Administração em Saúde Pública e Administração Hospitalar.

A partir da análise de dados foram construídas categorias para descrever a temática, sendo elas: Orientar e se (re)adequar: evitando complicações; Educação para o cuidado com os pés e com a pele; Orientações quanto ao uso dos fármacos; Cuidados com a alimentação e Educação em saúde com os familiares. No estudo os enfermeiros consideram a prática de educação em saúde no hospital como importante e, o processo educativo dos enfermeiros realizado contemplava ações que abrangeram as pessoas com a diabetes e familiares.

\section{Orientar e se (re)adequar: evitando complicações}

Para os enfermeiros, participantes do estudo, orientar as pessoas quanto aos sinais e sintomas e, o que a diabetes poderá ocasionar enquanto complicações faz parte da educação em saúde. Essas orientações versaram, por exemplo, sobre a perda da visão e lesões nos pés em decorrência do aumento da glicose no corpo. Também contemplaram os cuidados com a administração de insulina. Para isso, os enfermeiros relataram utilizar linguagem acessível, clara e de fácil entendimento. Ainda, descreveram que foi necessário adequar o "vocabulário" ao do paciente, uma vez que, descreveram que há diferenças socioculturais entre um paciente e outro, como evidencia-se nos relatos:

Eu sempre tento orientar eles e fazer com que eles entendam que eles têm ou uma diminuição de produção de insulina ou uma não produção dessa insulina e tento orientar eles dentro do vocabulário deles, o quão importante é eles controlarem pra que eles não tenham outros problemas. Então eu tento assim, da melhor forma possivel, da maneira mais simples possivel explicar porque que isso acontece, o que, quais são os cuidados que tem que ter, e quais são as consequências de não cuidar dessa glicose porque a glicose é uma coisa que as pessoas não levam muito a sério (...). [E02]

$E$ [oriento] ficar atento a qualquer alteração do seu corpo, seja na madrugada, quando ele tiver alguma alteração. Enfim, são vários questionamentos e orientações que a gente dá, vai depender muito do paciente em si, da orientação dele também. [E04]

[...] cuidado com os pés, em função de se eles usam insulina ou não, a dieta, a parte da visão, muitos deles se queixam que já tem a função da retinopatia diabética. [E011] 
Os enfermeiros compreendem a importância de orientar as pessoas com diabetes no decorrer da hospitalização quanto a possíveis alterações que possam vir a apresentar em decorrência da diabetes. Para isso, se utilizam do diálogo para compartilhar informações com vistas à prevenção de complicações.

\section{Educação para o cuidado com os pés e com a pele}

Os enfermeiros relataram educar pacientes quanto aos cuidados com os pés. Destacaram a importância dos pacientes observarem seus pés e identificarem sinais e sintomas, como a diminuição da sensibilidade, circulação, presença de calos e/ou lesão. Também forneceram informações sobre a limpeza/higiene e cuidado dos pés, além de reforçarem a importância de protegê-los mediante o uso de meias e calçados confortáveis durante a internação hospitalar, evitando, assim, quedas e lesões. Em decorrência de experiência prévia de alguns enfermeiros na atenção básica, esse cenário foi apontado como espaço relevante e de destaque para desenvolver a educação em saúde.

$O$ uso de calçados adequados para deambular no hospital, pedir para que usem calçados fechados, isso já é feito como rotina (...). [E05]

Eu trabalho também em saúde pública que a gente falava muito, muito em função dos cuidados com os pés, então tu vem para unidade de hospitalar, automaticamente tu pensa nos pés deles, então ai, em função de ver a circulação, de ver as unhas, de orientar quanto a isso, né.[E011]

Sempre orientar eles que tomem bastante cuidado que eles não batam os pés nas camas ou nas cadeiras quando eles vão deambular, ter cuidado na hora do banho também, quando eles vão ao banheiro. Então essas coisas assim, cuidado com as extremidades principalmente, porque o paciente diabético tem um risco muito grande de se machucar e nem perceber, porque eles acabam não sentindo as extremidades, geralmente é a coisa que eu mais faço assim né, tipo de orientar eles quanto a esses cuidados assim que tem que ter. [E014]

Em relação à pele, os enfermeiros acentuaram a orientação quanto à utilização de hidratantes com a finalidade de fortalecêla e evitar lesões cutâneas. Esses hidratantes foram fornecidos por eles para uso durante a hospitalização. Outras orientações corresponderam ao estímulo para a deambulação aos pacientes com condições de fazê-la. Já, para os que possuíam dificuldades de mobilidades deram ênfase ao preparo do leito, orientando para manter os lençóis sem dobras para evitar atrito com a pele, usar travesseiros e/ou cobertor para manter membros inferiores elevados, com o objetivo de estimular a circulação e evitar o surgimento de lesão por pressão. Esses achados podem ser identificados nos relatos a seguir:

[...] se o paciente deambula, a gente pede pra eles caminharem mais, né, pra ele, pra circulação melhorar, que eles podem fazer aqui dentro do hospital, tomar banho, secar bem entre os dedos dos pés, secar bem as unhas, ter cuidados, usar meias confortável, meia que não corte a circulação. E também que os lençóis sejam esticados para não ter atrito, não ter prega, essas coisas assim, manter elevado quando possivel, um travesseiro, um cobertor embaixo para não ficar encostando tanto o calcâneo principalmente, são cuidados que a gente pode orientar enquanto estão aqui, para eles fazerem aqui. [E011]

A gente também cuida a questão de lesões, porque tem a função da cicatrização que fica prejudicada, então a gente orienta, não usar aquele chinelo de dedo porque às vezes tropeça, machuca. A hidratação da pele que a gente fornece os [nome do creme hidratante], o que for necessário para fortalecer essa pele. [E012]

Os enfermeiros reconheceram o cuidado com os pés e com a pele dentre as ações para desenvolver a educação em saúde no hospital. Dessa forma, destacaram a importância de fornecer orientações voltadas preferencialmente à prevenir 
complicações nos pés e pele.

\section{Orientações quanto ao uso dos fármacos}

Os enfermeiros demonstraram preocupação quanto ao uso de medicamentos pelas pessoas com diabetes durante a hospitalização. Essa preocupação esteve relacionada ao tipo de medicamento, à quantidade e ao horário de uso. Apontaram, ainda, a importância de orientar ao paciente o horário dos medicamentos, favorecendo a interação e oportunizando espaço para o esclarecimento de dúvidas. A vigilância quanto à administração de medicamentos oriundos somente do hospital também foi mencionada pelos participantes. Tais achados são evidenciados nos seguintes excertos:

Eu acho importante o paciente saber o horário da administração dos medicamentos, o horário que acontece aqui dentro porque dai tu podes cobrar, ou perguntar, ou tirar alguma dúvida a respeito, interagir junto com a equipe, isso eu acho importante. [E010]

Mas aqui para nós é tranquilo né, a gente cuida se eles estão só utilizando as medicações que estão sendo dadas aqui. Tem muitos pacientes que, às vezes, vêm, já estão acostumados a usar aquela medicação em casa. A gente já olha nas gavetas com a permissão deles para ver se não tão associando a medicação deles com a nossa, a gente sempre observa, o que que tem em cima daquela mesinha ali, lateral, às vezes tem essa brincadeira né, "ah, mas não é meu, é da minha filha, é da acompanhante", mas a gente fica observando, a gente sabe quem são aqueles pacientes, que não respeitam muito o tratamento. [E015]

Para os pacientes com alta hospitalar os enfermeiros utilizaram uma linguagem de fácil entendimento para orientá-los e guiá-los no uso das medicações diárias no domicílio. Entre elas, a identificação, o uso de símbolos que representam o dia e a noite, sol e lua, respectivamente, como facilitadores para associar ao horário e turno que devem fazer uso dos medicamentos. Esses achados podem ser identificados no seguinte relato:

Nós colocamos as identificações quando precisa fazer a medicação, qual éo horário que é, ou se é, a mesma coisa que eu fazia na atenção básica, eu cheguei já a fazer aqui, de colocar sol e lua, sabe? [E05]

Outra orientação identificada nos diálogos dos enfermeiros foi fornecer informações quanto à insulina. Eles relataram que é necessário orientar previamente quanto à finalidade, os efeitos que podem surgir em decorrência do uso, como armazenar, quais os locais de aplicação, a forma de administração, a quantidade a ser administrada e a importância da utilização nos horários prescritos. As orientações tiveram a finalidade de promover o conhecimento e esclarecer dúvidas e, foram intensificadas quando os pacientes estavam com a previsão de alta hospitalar. Isso pode ser evidenciado nos seguintes relatos:

A dificuldade das próprias medicações a gente tenta orientar muito porque, porque muitos deles, ou nunca fez ou quando já fez, tanto diabete tipo 1 como 2, eles têm dificuldade de entender o que é insulina e pra que serve, a gente explica muito porque eles passam mal também às vezes, tem hipoglicemia por conta da própria quantidade de insulina, que a insulina precisa ser ajustada várias vezes, né. (...) Então a gente orienta tudo isso, orienta insulina, onde é que ele vai deixar, quantos graus tem que estar no ambiente para deixar aquilo exposto, então a gente dá toda essa orientação para ele ir embora [alta hospitalar]. [E03]

Fica muito a responsabilidade hoje de enfermagem, administração da insulina, como fazer, como eles [pacientes] fazem diretamente, os locais de aplicação, a quantidade. [E08]

Orientação em relação à forma de administração, que é muito assim, os pacientes, eles não sabem como administrar a insulina. Eles não sabem como armazenar a insulina. (...) Então eles não sabem como acondiciona, eles não sabem como aspirar, eles não sabem como 
administrar, a homogeneização, nada disso eles sabem, o tempo que tem que aguardar antes de retirar a agulha, nada disso os pacientes sabem, né, são raros os pacientes na verdade que sabem, né. Os melhores locais de administração. (...) Locais de administração, a importância dá, do uso do hipoglicemiante oral, né, que tem muito que, "ah, hoje eu não vou fazer, vou comer doce, não vou usar", como se anulasse o efeito, então são orientações nesse sentido né, tentando dá mais autonomia pra eles né, em busca de seu próprio cuidado né. [E09]

Conforme tu vê assim, é conforme é o paciente né, que tem pacientes que já fazem, usam muito tempo, ai tu pergunta, "ah mas quanto faz?", às vezes até pede pra mostrar quanto eles fazem né, porque as vezes eles dizem que é 4 mas na hora ali eles não tão mostrando o 4 na seringa. Né, porque a seringa é mais complicadinho, cada pontinho é duas unidades então tudo depende do paciente pra ti dar as orientações, tem uns que realmente sabem bem né. [E011]

Para os enfermeiros o rodízio da administração da insulina é um dos cuidados necessários a fím de evitar complicações, como abcesso e edema. Para eles é uma orientação e prática que as pessoas com diabetes têm resistência a fazer e seguir. Um dos motivos dessa resistência é justificado pelos pacientes, como sendo locais de maior sensação dolorosa. Relataram ainda que as orientações são dadas de acordo com cada paciente e suas particularidades. Identifica-se isso no seguinte relato:

Está fazendo [insulina] sempre no abdômen, por que está fazendo sempre no abdômen? Às vezes eles [pacientes] são resistentes. Ah, enfermeira porque aqui eu sinto menos dor. Mas tu já tentaste aqui no antebraço, nessa gordurinha no braço? Aqui na pochetezinha, aqui na coxa? "Ah, não enfermeira, eu já tentei e realmente dói muito". Então tá bem, então faz no abdômen, mas com o rodízio no abdômen, no quadrante superior direito, esquerdo e aí vai rolando em torno da região umbilical, dois dedos, e aí a gente vai dando essas orientações. Porque eles também têm que saber do cuidado deles, porque daqui a pouco eles vão para casa e eles vão ter que continuar, eles vão ser diabéticos, eles vão ter que fazer insulina em casa, então eles também tem que entender porque que a enfermagem faz esse rodizio. A gente vai lá e explica a função de toda hora estar picando no mesmo local, daqui a pouco faz um edema, tá com um abcesso pode até acontecer, ai a gente explica tudo para o paciente das orientações. [E012]

Frente ao exposto, apreende-se que o enfermeiro desenvolve, em sua prática no hospital, ações com a finalidade de educar e monitorar o paciente em uso de hipoglicemiantes e insulinoterapia. Ao educar dá ênfase à importância de o paciente seguir o horário dos medicamentos, demonstrar a administração da insulina, o esquema de rodízio e locais para a aplicação, assim como deve ser realizada e as possíveis complicações que podem ocorrer quando o uso inadequado. Além disso, o enfermeiro o informa sobre o armazenamento e, conservação quando no domicílio.

\section{Cuidados com a alimentação}

No que se refere à alimentação, os enfermeiros referiram educar os pacientes sobre o consumo exclusivo de alimentos ofertados pelo hospital. As orientações descritas pelos participantes visavam a promoção de uma "alimentação saudável", considerando que os pacientes demonstram conhecimento insuficiente sobre o que se pode ou não comer. Os enfermeiros reforçaram, ainda, que as orientações são realizadas conforme cada paciente e suas necessidades. Tais achados podem ser identificados nos seguintes excertos:

Tem que ficar cuidando da dieta. Por exemplo, paciente pós-cirúrgico ele tem que ter uma dieta adequada, ele tem que manter a glicemia e, aí muitas vezes a gente vê que eles não têm, recebem dieta de casa, coisas desse tipo. Ou eles mesmos descem, como aqui tem a lancheria, a lancheria aqui embaixo da Santa Casa, eles 
descem ali e pegam, compram alguma coisa. [E01]

[...] a única coisa que eu geralmente peço assim, mas na verdade para quase todos os pacientes, mas para os diabéticos eu peço em especial é para eles [familiares] não trazerem comida de casa, para eles comerem só o que está vindo do hospital, para gente ter um controle maior $e$ melhorar o HGT deles, eu sei que não é para ninguém trazer comida de casa, mas alguns trazem, e ai a gente sempre pede para eles não trazerem. [E013]

Uma das grandes dúvidas que eu vejo deles, acho que quase todos que chegam aqui, acho que não é nem tanto da medicação e dos cuidados, é da alimentação. Porque, às vezes, a gente diz assim, não, não come esse pão porque a farinha é branca. E eles dizem "não, mas ninguém nunca me falou que não podia comer esse pão". [E03]

Então, assim, dentro da unidade que eu mais utilizo, a informação que mais passo, principalmente quando ele já internou é que só coma a comida do hospital, porque tem a questão toda dos hábitos alimentares que os pacientes descompensam, às vezes. Porque tem toda uma situação de causa social, de, às vezes não é o paciente que faz a comida em casa, é o familiar, é toda uma questão de vínculos e social, de estrutura do familiar, do paciente. Então, a gente sempre pede que coma somente a comida do hospital enquanto está aqui, porque a gente demora às vezes para perceber todos os hábitos do paciente. [E05]

Alguns enfermeiros solicitam apoio da equipe de nutrição do hospital, para avaliação e orientação, principalmente em casos de pacientes com maior dificuldade de entendimento ou aqueles que moram sozinhos. Isso pode ser identificado nas seguintes falas:

Quando eu vejo que tem alguma dúvida, ou o paciente é um pouco confuso ou mora sozinho, tem alguma dificuldade em casa, eu sempre chamo o pessoal da nutrição. [...] só quando percebo que eles estão usando uma outra dieta, que não seja a dieta do hospital, mas também sempre peço ajuda para nutrição pra fazer essa abordagem. [E015]

Eu fico bem feliz com o outro lado, que é a parte nutricional, sempre tem uma orientação da nutrição (...) [E02]

Nesta categoria foi possível identificar que cuidar da dieta e dos alimentos consumidos pelos pacientes no hospital é uma das ações que representam ser um desafio ao enfermeiro considerando sua importância no controle e monitoramento da glicemia capilar. Para isso, por vezes, contam com a equipe de nutrição do hospital para abordar e orientar os pacientes.

\section{Educação em saúde com os familiares}

Nas falas foram identificadas ações de educação em saúde voltadas aos familiares. Elas diziam respeito à importância do cuidado com o paciente. Os enfermeiros destacaram que quando os familiares já foram orientados anteriormente o cuidado se torna mais fácil, pois pressupõem que eles têm conhecimento e entendimento sobre os cuidados a serem desenvolvidos durante a hospitalização e posteriormente no domicílio. Relatou-se que os familiares, por vezes, precisam ser incluídos no cuidado hospitalar. Por isso, os enfermeiros buscaram interagir, dialogar e construir vínculos com eles, inserindo-os nas ações de educação em saúde. Esses achados são constatados no seguinte excerto:

Normalmente a gente também na hora, quando tu identificas alguma alteração, alguma coisa, tu orientas, reorienta esse familiar né, falar da importância do cuidado, da atenção como o paciente né, normalmente é isso. Eles já tem uma orientação prévia né, então eu acho que isso já facilita o cuidado, porque muitas vezes a gente acaba não prestando tanto, dispensando tanta atenção em relação a isso, porque quando eles chegam aqui eles já dizem: "é diabético, usa metformina, usa glibenclamida, usa insulina, o paciente é isso é aquilo", eles já têm uma vivência 
prévia disso né, tu pressupõe que eles já tenham conhecimento, né? [E014]

Contudo, evidenciou-se nos diálogos dos enfermeiros que, às vezes, o entendimento da "família é difícil", então orientaram quanto ao uso de alimentos como aqueles que são "doces" e que "não são doces", para explicar que ambos contêm açúcar, assim como os carboidratos. Saber o que é um açúcar e diferenciar um alimento de outro, para os enfermeiros, foi considerado como fundamental no preparo e escolha dos alimentos a serem utilizados nas refeições e, consequentemente, auxiliar as pessoas no controle da glicemia, seja no domicílio ou durante a hospitalização. Um aspecto de maior preocupação para os participantes referente à alimentação correspondeu aos alimentos trazidos de casa pelos familiares. Esses alimentos, para eles, são os responsáveis pelo difícil manejo do tratamento, consequentemente complicações, e maior tempo de internação das pessoas no hospital.

A gente nota que tem familias que, às vezes até o entendimento da própria famillia é difícil, às vezes as orientações que dou, a comida, a nutrição, é mais assim para família, para explicar que certos alimentos têm muito açúcar. Assim, para eles entenderem porque eu acho que o diagnóstico do diabetes, o diagnóstico, eu com a minha experiência na UBS era o diagnóstico mais difícil que eu tinha pra dar, porque as pessoas achavam assim, achavam não né, entendiam que iam ter que mudar toda comida e nunca mais iam poder comer doce, nunca mais, e de certa forma sim, mas outras comidas que não são doces também tem grande quantidade de açúcar que elas teriam que reduzir e elas não entendiam isso porque a comida não é doce né, o arroz, a farinha né, tudo isso que tem carboidratos complexos, e eles não entendem isso como açúcar, eles entendem isso como comida de sal, então isso tudo é muito

dificil pra tu dizer pra pessoa e aqui no hospital algumas pessoas a gente nota que ainda não conseguem compreender completamente o diagnóstico, outas não, ou já fazem isso há muitos anos né, outras já lidam bem com isso (...) [E013]

$A$ alimentação tem que tá falando, "olha procura comer o que vem lá, do hospital”, porque a gente tem a nutrição bem ativa aqui no hospital. Tu comes o que tá no hospital porque a gente tá fazendo o teu controle da glicemia e daqui a pouco trazem [o familiar] um pudim de casa e alguma coisa e, ai o pessoal da endócrino fica sem entender. [E012]

Nessa categoria apresentou-se a interação do enfermeiro com a família da pessoa com diabetes. Nessa interação os profissionais descrevem sua vivência e apontam para a influência da família no cuidado de seu familiar no hospital e que por vezes se apresenta como um obstáculo para a equipe de enfermagem.

\section{Discussão}

Neste estudo, as ações de educação em saúde desenvolvidas pelos enfermeiros à pessoa com diabetes durante a hospitalização contemplaram um processo de ensino-aprendizagem com ênfase em orientações quanto à prevenção de complicações. Os enfermeiros que participaram da pesquisa compreendem e reconhecem como importante a prática da educação em saúde às pessoas com diabetes no hospital.

A atividade de educação em saúde foi descrita como paralela ao processo de assistência. $\mathrm{O}$ mesmo foi identificado em estudo, que não há momento específico para ensinar, e sim o enfermeiro necessita considerar desde o momento que ingressa no hospital e detecta as necessidades ${ }^{13}$. Contudo, estudo aponta que o momento de realização do histórico de enfermagem é o momento ideal para iniciar o cuidado educativo ${ }^{7}$.

Além disso, as orientações caracterizaram-se de forma prescritiva em que a posição da pessoa com diabetes estava direcionada a cumprir as orientações/prescrição. Diante disso, a 
ênfase das orientações esteve centrada no comportamento da pessoa e na valorização da sua dimensão biológica como eixo central.

Essa forma prescritiva na abordagem do profissional de saúde descreve o comportamento da pessoa doente em termos de cumprimento, em que é vista como problemática e desobediente, e que o sucesso do tratamento depende exclusivamente dela seguir a prescrição médica e de enfermagem. Esse enfoque biomédico não considera as experiências e práticas subjetivas na perspectiva das pessoas que vivenciam o adoecimento ${ }^{19}$, o que pode dificultar o processo de cuidado e autocuidado e realização do tratamento. Achados semelhantes em estudo identificaram que enfermeiros replicam um modelo de educação voltado para práticas higienistas, em que o conhecimento é transmitido de forma vertical, ignorando a integração e articulação de diferentes saberes e práticas ${ }^{12}$.

A educação em saúde tem sido desenvolvida, predominantemente, sob práticas higienistas, palestras prescritivas de hábitos, comportamentos e condutas, considerando os indivíduos de forma passiva, com ênfase em conhecimentos técnicos sobre a doença e como cuidar da saúde, sem considerar o saber popular e o contexto de vida dos indivíduos ${ }^{20}$. A valorização do corpo biológico permeando a conduta dos enfermeiros em detrimento à compreensão do ser humano enquanto ser biopsicossocioespiritual é um dos achados em estudo com enfermeiros, apontando para a necessidade de transformar a prática de enfermagem no hospital ${ }^{12}$. Contudo, na educação em saúde é importante a valorização dos saberes e conhecimento dos indivíduos e, não somente o conhecimento científico $^{21}$.

Ainda sobre esse aspecto, estudo realizado com enfermeiros da atenção primária em saúde identificou resultados positivos após o desenvolvimento de ações de educação em saúde, por meio do controle da glicemia e dos relatos dos pacientes sobre à mudança de hábitos. Porém, os enfermeiros relacionam a baixa participação das pessoas com diabetes nas atividades oferecidas de educação em saúde, ao modo como as elas são organizadas e ofertadas, ao desconhecimento das pessoas sobre a doença e a cultura dos pacientes que, de forma geral, valorizam as ações compatíveis ao modelo biomédico ${ }^{22}$.

As orientações foram realizadas pelos enfermeiros à pessoa com diabetes de forma individual e/ou com os familiares de forma coletiva nas enfermarias. Ações em grupo tanto com seus pares, quanto de familiares, não foram identificadas no cenário das unidades de internação do hospital, assim como não se evidenciou o uso de materiais didáticos para tais orientações. Evidências na literatura apontam que orientações educativas em grupos de pessoas com diabetes, são possíveis de serem realizadas no hospital, além de integrar seus familiares, esse pode ser um espaço de construção de conhecimento, trocas e compartilhamento de experiências entre $\mathrm{si}^{7}$. Nesse espaço, enfatiza-se a importância de o enfermeiro utilizar material didático, de maneira lúdica e educativa, na forma de vídeos ou fôlderes nas ações. Além disso, as atividades em grupo devem conter a colaboração de profissionais de saúde de diversas áreas multiprofissionais de saúde ${ }^{7}$ Uma educação em saúde efetiva é aquela em que a intervenção educativa se baseia em problemas reais, ou seja, na realidade e cotidiano do indivíduo com a finalidade de aplicabilidade do conteúdo apreendido, sendo um exemplo dessa abordagem as ações em grupo ${ }^{23}$. A intervenção em grupo se apresenta como necessária para o comportamento de prevenção e autocuidado, além de promover maior dissociação das informações, aprendizagem mútua, interação social e apoio emocional $^{23}$.

O hospital foi descrito como um espaço para capacitar a pessoa com diabetes e familiares. Essa ação esteve voltada, como 
maior ênfase, sobre o uso da insulina, sendo o momento da administração propício para a ação e aprendizado. A participação da pessoa com diabetes e familiares na assistência possibilita que os mesmos se tornem protagonistas no processo de cuidado, garantindo práticas seguras para o autocuidado no domicílio. O ensinar é descrito como uma ação que ocorre com a conexão entre enfermeiro e paciente em que ao mesmo tempo se realiza um cuidado e, se ensina a realizar o próprio cuidado no domicílio $^{13}$. A dificuldade na autoaplicação da insulina no domicílio foi identificada em estudo como um obstáculo na continuidade e adesão ao tratamento para diabetes ${ }^{24}$. Diante disso, se faz necessário o deslocamento do paciente e/ou familiar da posição de observador, pois dessa forma se sentirão mais seguros para realizar o tratamento no domicílio após a alta hospitalar?

Nesse sentido, estudo de revisão sistemática avaliou a eficácia do uso do método "Teach-back" - aquele em que o paciente confirma as informações recebidas ao profissional, de modo a garantir a efetividade da comunicação - na aderência ao tratamento e autocuidado de pessoas com doenças crônicas. Neste, verificou-se que quatro estudos confirmaram a melhoria do conhecimento específico da doença nos participantes, um estudo mostrou melhora estatisticamente significativa na adesão à medicação e dieta entre os pacientes com diabetes, dois estudos encontraram melhorias estatisticamente significativas na autoeficácia, cinco estudos encontraram uma redução nas taxas de readmissão e hospitalização, mas nem sempre foram estatisticamente significativas, dois estudos mostraram melhora na adesão à dieta, exercícios e cuidados com os pés entre as pessoas com diabetes ${ }^{25}$.

Ações de educação em saúde podem capacitar indivíduos, familiares ou grupos, com a finalidade de contribuir na melhoria das condições de vida, bem como estimular a reflexão crítica sobre suas necessidades de saúde e ações requeridas para sua resolução ${ }^{12,26}$. Por esse motivo, a criação de vínculo é necessária, com vistas a aproximar os diferentes sujeitos envolvidos no cuidado, inclusive o familiar, frequentemente esquecido na assistência hospitalar, mas sinalizado pelos sujeitos como importante estratégia que potencializa a educação em saúde nesse cenário ${ }^{12}$.

O conhecimento do estado de saúde e dos meios que podem ser utilizados para dar continuidade ao cuidado no domicílio é fundamental para que a pessoa com diabetes consiga realizar o tratamento, evitar complicações e manter a qualidade de vida. Estudos apontam que as pessoas com diabetes possuem déficit de conhecimento sobre a doença e tratamento comprometendo o autocuidado ${ }^{11,24}$. Associado ao nível de conhecimento, o nível de letramento inadequado influencia negativamente a pessoa de realizar o autocuidado, principalmente em seguir uma dieta saudável, orientação alimentar e avaliação de glicemia ${ }^{27}$. Pessoas com níveis de escolaridade baixos apresentam maiores dificuldades para compreender e aderir ao tratamento, o nível de escolaridade demonstra influência frente ao aprendizado com relação ao autocuidado, visto que os pacientes apresentam conhecimento em relação a diabetes causar complicações, mas não entendem seu conceito e não sabem identificar tais complicações com precisão ${ }^{28}$. Há evidências na literatura que quanto maior o nível de conhecimento maior será a qualidade de vida e autonomia sobre o tratamento ${ }^{29}$.

A educação em saúde individual tem como instrumento potencial o cuidado ao indivíduo. Assim, a intervenção educativa nesse modo leva à forma de individualizar o cuidado, abranger um único indivíduo e uma formação de vínculo maior ${ }^{23}$. Informações sobre alimentação, cuidado com os pés e glicemia capilar proporcionada pelos enfermeiros como processo social centrado na pessoa com diabetes, tem como objetivo sensibilizar e estimular para o autocuidado no domicílio. Para ambos, pessoa com diabetes e 
familiares, informações com a finalidade de ajuda a fomentar sua participação e a tomar decisões sobre a atenção ${ }^{13}$. Nesse contexto, as orientações, além de informar sobre a doença, ajudam os pacientes a reconhecerem os sinais e sintomas de alterações do nível glicêmico, tanto relacionado à alimentação, quanto a outros fatores. Além disso, os efeitos colaterais de medicamentos utilizados, pois as circunstâncias que ocorrem antes e após a administração podem acarretar eventos adversos e o paciente precisa estar ciente ${ }^{11}$. A educação em saúde teve como foco orientar pacientes e familiares, com maior ênfase, no cuidado com pés, pele, alimentação e uso de medicamentos. Os resultados do presente estudo indicam que os enfermeiros procuram adequar as orientações a uma linguagem de "fácil entendimento", para as pessoas com diabetes, principalmente para aqueles que possuem um menor nível socioeconômico e de letramento. $\mathrm{O}$ tratamento não farmacológico, hábitos saudáveis de alimentação e exercício físico, foram identificados como atrito para os pacientes ${ }^{24}$.

Entre os motivos para não seguir com o tratamento está relacionado a questão financeira como um impedimento para seguir as orientações fornecidas, principalmente quando se fala em alimentação saudável ${ }^{24}$. $\mathrm{Na}$ literatura é descrito sobre a necessidade do profissional de saúde utilizar uma linguagem neutra e sem julgamentos, para evitar que a pessoa que está ouvindo crie uma percepção diferente com relação à doença e, se sinta colocado em posição de sentenciamento. Com isso, pode haver um afastamento social do paciente em relação à sua rede de apoio por vergonha, culpa e sentimento de fracasso, assim como, pode-se considerar "um fardo" para o sistema de saúde ${ }^{30}$.

Estudo aponta que uma das ferramentas para desenvolver educação em saúde são os mapas de conversação. Eles são utilizados como uma estratégia educativa pelos profissionais de saúde ${ }^{14,23}$.
Esse instrumento tem se mostrado eficaz internacionalmente para o autocuidado de pessoas com diabetes. Ao utilizá-lo em grupo, em um ambulatório, possibilitou às pessoas com diabetes, além de compartilhar experiências, identificar a escassez de conhecimento no que diz respeito a quais alimentos podem consumir, assim como quantidades e qualidade para uma refeição nutritiva e, que não resulte em complicações futuras ${ }^{14}$.

Enfatiza-se que os enfermeiros utilizem o diálogo como ferramenta para sensibilizar o paciente com diabetes a construir o próprio conhecimento de forma gradativa, que será mais significativo e duradouro para o autocuidado da doença e tratamento de forma autônoma. Diante disso, o enfermeiro tem papel fundamental para proporcionar orientações que motivem e mobilizem as pessoas com diabetes para realizar o autocuidado. Dessa forma, entende-se que a comunicação é construída e utilizada pelos enfermeiros como um aspecto de fundamental importância para paciente e seus familiares, considerando o processo de autocuidado, pois ela poderá influenciar na percepção da pessoa com diabetes no decorrer do adoecimento. Uma comunicação ineficaz poderá ocasionar estresse e desânimo em relação à doença e ao tratamento, que associado ao medo e culpabilização por não ter "seguido com as orientações e cuidados", poderá resultar em uma diminuição na procura por atendimento nos serviços de saúde e consequentemente a levará, de forma mais agressiva, a desenvolver complicações.

\section{Conclusão}

As ações de educação em saúde à pessoa com diabetes desenvolvidas pelos enfermeiros na hospitalização foram orientações individuais e coletivas, para a pessoa com diabetes mellitus e o familiar. Essas abrangeram a temática da doença, ou seja, o que é a diabetes, quais os sinais, sintomas e complicações que podem ser acarretadas. Também buscaram ensinar 
para que serve a insulina no contexto da diabetes e como se dá sua administração.

A educação em saúde é vista como a informação fornecida aos pacientes e familiares pelos profissionais de saúde, contribuindo para a prevenção de complicações e manutenção da saúde. $\mathrm{O}$ hospital é um dos espaços de cuidado à saúde em que o paciente permanece de forma integral, assim é necessário estimular e investir em ações que capacitem e qualifiquem a equipe de enfermagem, e que esse tema seja incluído nas atividades da instituição como parte da educação continuada e permanente.

Destaca-se a importância da educação para o paciente, pois em seu domicílio será necessário dar continuidade ao tratamento. Diante disso, é necessário que o profissional considere o paciente em sua condição emocional, social, econômica e cultural, uma vez que, está inserido em um contexto que pode influenciar no sucesso do tratamento e, consequentemente na qualidade de vida considerando o viver com uma doença crônica.

Em relação à pesquisa, é desejável complementar essa perspectiva com as vozes de outros atores, como gestores, familiares e outros profissionais de saúde. Como limitação, a técnica de coleta utilizada foi a entrevista semiestruturada, o que significa que aqui a voz dos participantes foi priorizada em relação a outros elementos que possibilitariam dar informações para melhor compreensão da educação em saúde, como, por exemplo, a observação participante e, articular e indagar se as falas correspondem às práticas no hospital.

\section{Referências}

1. Whiting DR, Guariguata L, Weil C, Shaw J. IDF diabetes atlas: global estimates of the prevalence of diabetes for 2011 and 2030. Diabetes Res Clin Pract. 2011; 94:311-21.

2. Federação Internacional de Diabetes. IDF Diabetes Atlas, $8^{\mathrm{a}}$ ed. Bruxelas, Bélgica: 2017. Disponível em: https://diabetesatlas.org/upload/resources/previous/files/8/IDF_DA_8eEN-final.pdf

3. Sociedade Brasileira de Diabetes. Diretrizes da sociedade brasileira de diabetes 2019-2020. São Paulo: Editora Clannad; 2019.

4. Datasus [base de dados na Internet]. Brasília: Ministério da Saúde [acesso em jul 2020]. Disponível em: http://tabnet.datasus.gov.br/cgi/tabcgi.exe?sih/cnv/niuf.def

5. Kaya Z, Karaca A. Evaluation of Nurses' Knowledge Levels of Diabetic Foot Care Management. Nurs Res Pract. 2018; 2018:8549567.

6. Signor F, Leguisamo CP, De Marchi ACB, Bavaresco SS, De Oliveira LZ, Pillatt AP. Conhecimento e educação em saúde de idosos portadores de diabetes mellitus. Fisioter Bras. 2016; 17:171-5.

7. Arruda C, Silva DMGV. A hospitalização como espaço para educação em saúde às pessoas com diabetes mellitus. Rev Pesqui (Univ Fed Estado Rio J, Online). 2020; 12:37-45.

8. Marques MB, Coutinho JFV, Martins MC, Lopes MVO, Maia JC, Silva MJ. Educational intervention to promote self-care in older adults with diabetes mellitus. Rev Esc Enferm USP. 2019; 53:e03517. 
9. Ministério da Saúde; Secretaria Executiva; Secretaria de Gestão do Trabalho e da Educação na Saúde. Glossário temático: gestão do trabalho e da educação na saúde. Brasília: Ministério da Saúde, 2012.

10. Pelicioni MCF, Pelicioni AF. Educação e promoção da saúde: uma retrospectiva histórica. Mundo Saúde. 2007; 31:320-8.

11. Souza NPG, De Oliveira GYM, Girão ALA, Souza LM, Maniva SJCF, De Freitas CHA. Adoecimento por hipertensão arterial e Diabetes Mellitus: concepções de um grupo de pacientes hospitalizados. Rev enferm UERJ. 2015; 23:52-7.

12. Figueira AB, Amestoy SC, Cecagno D, Tristão FS, Trindade LL, Correa VA. Visão do enfermeiro frente à prática da educação em saúde no ambiente hospitalar. Cogitare Enferm. 2013; 18:310-6.

13. Castro-Meza AN, Pérez-Zumanob SE, Salcedo-Álvarezc RA. La enseñanza a pacientes con diabetes: significado para profesionales de enfermeira. Enferm univ. 2017; 14:39-46.

14. Carvalho SL, Ferreira MA, Medeiros JMP, Queiroga ACF, Moreira TR, Negreiros FDSF. Mapa de conversação: estratégia educativa no cuidado ao idoso com diabetes mellitus. Rev Bras Enferm. 2018; 71:925-9.

15. Minayo MCS. Amostragem e saturação em pesquisa qualitativa: consensos e controvérsias. Rev Pesqui Qual (Online). 2017; 5:1-12.

16. Bardin L. Análise de Conteúdo. São Paulo: Edições 70, 2016.

17. Conselho Nacional de Saúde (BR). Resolução n ${ }^{\circ} 66 / 2012$. Aprova normas regulamentadoras de pesquisas envolvendo seres humanos. Brasília; 2012.

18. Conselho Federal de Enfermagem (BR). Resolução no 564/2017. Aprova o novo Código de Ética dos Profissionais de Enfermagem. Brasília; 2017.

19. Conrad P. The Meaning of Medications: Another Look at Compliance. Soc Sci Med (1982).1985; 20:29-37.

20. Rigon AG, Neves ET. Educação em saúde e a atuação de enfermagem no contexto de unidades de internação hospitalar: o que tem sido ou há para ser dito? Texto Contexto Enferm. 2011; 20:812-7.

21. Falkenberg MB, Mendes TPL, De Moraes EP, Souza EM. Educação em saúde e educação na saúde: conceitos e implicações para a saúde coletiva. Ciênc Saúde Colet. 2014; 19:847-52.

22. Teston EF, Spigolon DN, Maran E, Santos AL, Matsuda LM, Marcon SS. Nurses' perspective on health education in Diabetes Mellitus Care. Rev Bras Enferm. 2018; $71: 2735-42$. 
23. Santos WP. Abordagens metodológicas utilizadas em intervenções educativas voltadas a indivíduos com diabetes mellitus. Enferm actual Costa Rica (Online). 2020; (38):260-71.

24. Reis P, Arruda GO, Nass EMA, Ratuchnei ES, Haddad MCFL, Marcon SS. Autocuidado e percepção do tratamento para o diabetes por pessoas em uso de insulina. Rev enferm UFSM. 2020; 10:1-20.

25. Ha Dinh TT, Bonner A, Clark R, Ramsbotham J, Hines S. The effectiveness of the teachback method on adherence and self-management in health education for people with chronic disease: a systematic review. JBI Database System Rev Implement Rep. 2016; $14: 210-47$.

26. Maciel MED. Educação em saúde: conceitos e propósitos. Cogitare enferm. 2009; 14:773-6.

27. Luz GOA, Alves DDA, Costa HKS, Da Silva Filho JC, Stratmann PF, Souza MAO, Santos ICRV. Associação entre o letramento funcional em saúde e o autocuidado com o diabetes mellitus. Cogitare enferm. 2019; 24:e66452.

28. Amaral RT, Barbosa AM, Teixeira CC, Brandão LGVA, Afonso TC, Bezerra ALQ, Tobias GC. Conhecimento dos diabéticos frente à doença e orientações no autocuidado. Rev enferm UFPE on line. 2019; 13:346-52.

29. Costa SS, Rosales RA, De Ávila JA, Pelzer MT, Lange C. Adesão de idosos com diabetes mellitus à terapêutica: revisão integrativa. Cogitare Enferm. 2017; 22: e47720.

30. Dickinson JK, Guzman SJ, Maryniuk MD, O’Brian CA, Kadohiro JK, Jackson RA, D'Hondt N, Montgomery B, Close KL, Funnell MM. The Use of Language in Diabetes Care and Education. Diabetes Educ. 2017; 43: 551-64.

\section{Como citar este artigo:}

Barcellos CRB, Zillmer JGV, Ramos BR, Barz DB, Cordeiro FR, Luiz MB. Educação em saúde à pessoa com diabetes mellitus no hospital . Rev. Aten. Saúde. 2021; 19(69): 103-117. 